\title{
Astromechanics of Dual Universe: A New Possible Explanation for the Universe Unsolved Problems
}

\author{
Mohammed B. Al-Fadhli ${ }^{1,2 *}$ \\ ${ }^{1}$ College of Science, University of Lincoln, Lincoln, LN6 7TS, UK. \\ ${ }^{2}$ Faculty of Engineering, University of Nottingham, Nottingham, NG7 2RD, UK. \\ *Correspondence: malfadhli@lincoln.ac.uk; mo.fadhli7@gmail.com; Tel.: +44-7866-206-238
}

\begin{abstract}
The necessity of the dark energy and dark matter in the present universe could be a consequence of the antimatter elimination assumption in the early universe. Current cosmological models that rely on the dark side have left many unsolved mysteries, remarkably: tension in Hubble parameter measurements, the accelerated expansion, the fast orbital speed of stars, the dark flow observations, cosmic horizon, space flatness, absent of the antimatter, etc. On the other hand, General Relativity (GR) has relied on the spacetime to demonstrate the movement of matter due to a local curvature caused by the presence of matter. Founded on this, I trace the evolution of the spacetime worldlines based on the evolution of the universe spatial scale factor and its evolution time in polar coordinates in order to construct a potential spatial curvature over the temporal dimension or a global spacetime curvature. The mathematical derivations of a positively curved universe governed by only gravity revealed two opposite solutions of the worldline evolution. This possibly implies that the matter and antimatter could be evolving in opposite directions as distinct sides of the universe. By implementing the derived model, we find a decelerated phase of spatial expansion during the first $\sim 10 \mathrm{Gyr}$, that is followed by a second phase of an accelerated expansion; potentially matching the tension in Hubble parameter measurements. In addition, the model predicts a final time-reversal phase of spatial contraction, due to rapid surge in density i.e. reversal entropy, leading to a Big Crunch of a cyclic universe. The predicted density is $\Omega_{0}=\sim 1.14>1$. Other predictions are (1) an evolvable curved spacetime at the decelerated phase that is transformed to flatness at the accelerated phase with internal voids which could continuously increase the matter and antimatter densities elsewhere in both sides. (2) the spatial curvature through time dimension along spacetime worldlines was found to increase galaxy orbital speed and (3) a calculable flow rate of the matter side towards the antimatter side at the accelerated phase; conceivably explaining the dark flow observation. These findings may indicate the existence of the antimatter as a distinct side, which influences the evolution of the universe instead of the dark energy or dark matter. These theoretical outcomes and predictions are promising, which can be verified, fine-tuned or disproved using astrometric data in future works.
\end{abstract}

Keywords: Accelerated Expansion, Space Flatness, Cosmic Horizon, Parallel Universe, Antimatter

\section{Introduction}

Spacetime is considered by GR as a four-dimensional manifold where its local curvature due to the presence of matter governs how the matter moves. However, no global spatial curvature over the evolution time or possible cosmic topology is currently theorized and tested, which may describe the matter movement through space over the evolution time of the universe. This could be an alternative approach and may provide physical reasoning for the accelerated expansion, the fast orbital star rotation, tension in Hubble parameter measurements, dark flow observations, cosmic horizon, current space flatness, the absent of the antimatter, etc. [1].

In addition, the fundamental CPT symmetry (Charge, Parity, and Time reversal) states that the matter and antimatter would have been created in the same quantities at the Big Bang [2]. In contrast, the matter-antimatter asymmetry, by the violation of the CP symmetry in the early era, could have given rise to today's matter-dominated universe according to the standard Big Bang theory [3]. 
However, advanced measurements of the fine structure of hydrogen and antihydrogen atoms were found to be consistent with the estimations of quantum electrodynamics theory [4], [5], which contradicts the $\mathrm{CP}$ violation assumption adopted in the standard Big Bang theory. Further, the monopole, horizon, and flatness problems arise from Big Bang singularity while the hypothetical theory of inflation to solve these problems was found to be problematic and unfalsifiable [6], [7].

As an alternative, the non-singular Big Bounce theory assumes the primordial substance was concentrated from a previous collapsed universe, and the universe experiences continuous expansions and contractions [8], [9]. However, a version of the Big Bounce theory could be recommended that a Bang of a primordial substance at thermal equilibrium produced a hot and dense early universe where the matter and antimatter of that fireball could have been separated by electromagnetic fields [10], [11]. In addition, according to the Big Bounce theory, the initial scale factor of the universe must be greater than zero [8], [9] where a constant $R_{i}$ could represent the initial space curvature. Besides, a closed finite universe could aid a large-scale cut-off in primaeval density fluctuations and may provide an agreement with low $\mathrm{CMB}$ anisotropy quadrupole observations [12]. Further, quantum entanglement could support closed universe that in which the energy that were at Big Bounce are always the same, therefore a cosmic conservation would ensure for example, the total spin of a pair of particles to be always conserved regardless to their locations.

To comply with the fundamental CPT symmetry and the laws of thermodynamics, cosmology and mathematical scientists have proposed that the universe could consist of matter and antimatter dominated halves/sides [3], [13], [14]. The first theory of a dual universe was proposed by Sakharov in 1967 [3], who proposed a baryonic asymmetry that may have violated the CP symmetry and created surplus matter. In addition, Petit in 1977 and Robles-Pérez in 2013 proposed a CPT preserved model with anti-universe of a reverse arrow of time [13], [14]. However, the dual universe theories appear to lack the comprehensive dual geometry and the evolution space curvature, which may allow them to provide physical explanations for the unsolved stated problems.

On the other hand, a few models adopting a single universe assumption were introduced prior to General Relativity. However, most of these models had a lot of uncertainty of how a finite Newtonian universe could survive the gravitational breakdown [15]. Einstein proposed the first cosmological model of a static universe in 1917 where he introduced the cosmological constant as the vacuum energy to balance gravity. De Sitter introduced an expanding and almost empty universe dominated by the cosmological constant in 1917. However, these modes were reported to be unstable [15]. In 1922, Friedmann developed an expansion-contraction model of the universe. The model proposed that the universe began from a small size and then expands to the critical scale factor, and then contracts towards the Big Crunch [16]. However, his model was ignored for some time as the expansion of the universe was not established until Hubble's discovery in 1929. In 1927, Lemaitre developed a cosmological model compatible with the Friedmann model and introduced the early state of the universe as a hot and dense period [15]. Nevertheless, after the discovery of the expansion of the universe, both De Sitter and Einstein jointly developed a simpler model of an expanding universe in 1932, where Einstein abandoned the cosmological constant [1], [17]. Furthermore, in 1998, the universe expansion was discovered to be accelerating [18]-[21], which invalidated the reliability of the available cosmological models. To end this, the current standard $\Lambda$ cold dark matter $(\Lambda \mathrm{CDM})$ model was developed, which proposed a flat expanding universe with dark energy as the vacuum energy, quintessence or cosmological constant to explain the accelerated expansion [22]. The $\Lambda \mathrm{CDM}$ model has provided appropriate alignments with astronomical observations [23]. However, the assumption of the dark energy continuous formation from vacuum to sustain the accelerated expansion is an obvious violation of the energy conservation law [24]. Also, the predicted value of the $\Lambda \mathrm{CDM}$ cosmological constant contradicts with the quantum field theory where the discrepancy between estimations and the experiment is about 120 orders of magnitude [25].

Similar to previous models, the $\Lambda \mathrm{CDM}$ model has faced inconsistency with the advancement of new astronomical observations [26], [27]. The recent Planck Legacy 2018 (PL18) release indicated the existence of an enhanced lensing amplitude in the CMB that is higher than what is expected in the $\Lambda \mathrm{CDM}$ model [28]. This endorses the existence of a positive curvature of the universe with a level of 
confidence greater than $99 \%$ [27]. In addition, the precise Hubble parameter measurements from the early universe using the Planck datasets based on the $\mathrm{CMB}$ show a lower value of expansion rate in comparison with the value of Hubble parameter in the present universe using the type Ia supernovae distance-redshift method [20], [26], where the variation is three standard deviations [26], [27]. Further, the cosmic shear observed by the Kilo Degree Survey 450 is conflicting with Planck datasets at about two standard deviations [27], [29]. Riess in 2020 found that the expansion of the universe is faster than what $\Lambda \mathrm{CDM}$ estimates where the disagreement between several independent measurements taken from the early and present universe is at four to six-sigma and concluded this outcome cannot be ignored [21]. Accordingly, a profound adjustment of the $\Lambda \mathrm{CDM}$ model or new physics are now growing due to this new evidence underlying the model assumptions [23]. On the other hand, several alternative theories which encounter the assumptions of the $\Lambda \mathrm{CDM}$ model are under consideration, such as modified gravity, entropic gravity, bimetric gravity, modified Newtonian dynamics, scale invariance of empty-space, large-scale variations in the matter density of the universe and the decaying dark matter [30]-[33].

In this paper, I trace the evolution of spacetime worldlines using basic cosmic model that correlates the universe spatial scale factor to its evolution time in order to construct a possible cosmic topology or a space curvature over evolution time. The paper is organized as follows: in Section 2, I modify the metric tensor to account for the initial space curvature according to the Big Bounce theory and derive the model. Sections 3, 4 and 5 discuss the stated problems. Section 6 summaries the conclusions. Finally, Section 7 presents future work.

\section{The Mathematical Model}

The Einstein-Hilbert action is given by:

$$
S=\int\left[\frac{c^{4}}{16 \pi G} R+L_{M}\right] \sqrt{-g} d^{4} x
$$

where $c$ is the speed of light in vacuum, $G$ is Newton's gravitational constant, $R$ is the scalar curvature, $L_{M}$ is the matter fields of the universe and $g$ is the determinant of the metric tensor $g_{u v}$. The integration of this action gives Einstein's field equations without a cosmological constant:

$$
T_{u v}=\frac{c^{4}}{8 \pi G}\left(R_{u v}-\frac{1}{2} R g_{u v}\right)
$$

where $T_{u v}$ is the energy-momentum tensor and $R_{u v}$ is Ricci curvature tensor [34]. The metric tensor $g_{u v}$ can be characterized using the Friedmann-Lemaitre-Robertson-Walker (FLRW) model [35], [36]. Based on equivalent Newtonian dynamics of this metric, the isotropic spherical coordinates of the FLRW metric could be enhanced to account for the initial space curvature as follows:

$$
d s^{2}=c^{2} d t^{2}-a^{2}(t)\left(\frac{d r^{2}}{1-k \frac{r^{2}}{R_{i}{ }^{2}}}+r^{2} d \theta^{2}+r^{2} \sin ^{2} \theta d \phi^{2}\right)
$$

where $d s$ is the four-dimension spacetime interval in polar coordinates, $a$ is the spatial scale factor, and $k \& R_{\mathrm{i}}{ }^{2}$ are constants representing the space curvature [34], [37] and the initial space curvature respectively. $k=1,-1,0$ for closed, open and flat universe while $R_{i}^{2}=1, \gtrsim 1, \lesssim 1$ for flat, positive and negative initial space curvature.

The tensor signature $(-,+,+,+)$ is utilised throughout this research. By solving the Ricci tensor for the enhanced metric tensor $g_{u v}$, we obtain its components:

$$
\begin{gathered}
R_{t}^{t}=3 \frac{\ddot{a}}{a} \\
R_{j}^{i}=\left(\frac{\ddot{a}}{a}+2 \frac{\dot{a}^{2}}{a^{2}}+2 \frac{k c^{2}}{a^{2} R_{\mathrm{i}}{ }^{2}}\right) \delta_{v}^{u}
\end{gathered}
$$


where $\delta_{v}^{u}$ is the Kronecker delta[34].

By solving the field equations in Eq (2) for a perfect fluid given by $T_{v}^{u}=\left(\rho+\frac{P}{c^{2}}\right) u^{u} u_{v}+\frac{P}{c^{2}} \delta_{v}^{u}$ and submitting the Ricci tensor components to them, we get the Friedmann equations that count for the initial space curvature:

$$
\begin{aligned}
& \frac{\dot{a}^{2}}{a^{2}}=\frac{8 \pi G \rho}{3}-\frac{k c^{2}}{a^{2} R_{i}{ }^{2}} \\
& \frac{\ddot{a}}{a}=-\frac{4 \pi G}{3}\left(\rho+3 \frac{P}{c^{2}}\right)
\end{aligned}
$$

where $\rho$ is the mass density and $P$ is the pressure. Using the notation $c=1$ and rewriting Eq (6) in terms of the conformal time $d \eta \equiv \frac{d t}{a}$ in its parametric form in the range of $(0<\eta<2 \pi)$ where $a_{0}$ can be normalized to 1 at time $t_{0}$ and $k=1$ by the definition of the metric for the positively curved universe [38]:

$$
\int d \eta=\int_{0}^{2 \pi} \frac{d a}{\sqrt{\left(2 \Omega_{0, m} a-\frac{1}{{R_{\mathrm{i}}}^{2}} a^{2}\right)}}
$$

where $\Omega_{0, m}=\frac{4}{3} \pi G \rho_{0}=\frac{\overline{\Omega_{0, m}}}{\overline{\Omega_{0, m}}-1}$ is the matter density and $\overline{\Omega_{0, m}}=\frac{\rho}{\rho_{\text {critical }}}$ is the matter density parameter. By integrating, we get the spatial scale factor of the universe as a function of the conformal time:

$$
a(\eta)=\frac{\Omega_{0, m} R_{i}^{2}}{2}\left(1-\cos \frac{\eta}{R_{i}}\right)
$$

In addition, we can obtain the evolution time of the universe $t(\eta)$ as a function of the conformal time using Eq (9) in terms of the Hubble parameter where $d t \equiv \frac{d a(\eta)}{H} \quad[38]$ :

$$
\int d t=\int \frac{\Omega_{0, m} R_{i}^{2}}{2 H}\left(1-\cos \frac{\eta}{R_{i}}\right) d \eta
$$

where $\frac{H^{2}}{H_{0}^{2}}=\frac{\Omega_{0}}{a^{3}}+\frac{\left(1-\Omega_{0}\right)}{a^{2}}$ and $\Omega_{0, m}=\frac{\overline{\Omega_{0, m}}}{\left(\Omega_{0, m}-1\right)^{3 / 2}}$ for a positively curved universe and is matterdominated [38]. By integrating, the evolution time of the universe is:

$$
t(\eta)=\frac{\Omega_{0, m} R_{i}^{2}}{2 H_{0}}\left(\eta-\sin \frac{\eta}{R_{i}}\right)
$$

Regarding the evolution of the matter density, according to the energy conservation $T_{; v}^{u v}=0$ and because of the isotropy, we get $\frac{\partial \rho}{\partial t}+3 \frac{\dot{a}}{\mathrm{a}} \rho+\frac{\dot{a}}{\mathrm{a}} T_{u}^{u}=0$ and $\frac{\partial \rho}{\partial t}+3(\rho+P) \frac{\dot{a}}{\mathrm{a}}=0$. This leads to $\rho a^{3}=$ constant for a matter-dominated universe [38] and by submitting Eq (9) to it, we get:

$$
\overline{\Omega_{m}}(\eta)=\frac{8 M_{1}}{\Omega_{0, m}{ }^{3} R_{i}^{6} \rho_{\text {critical }}}\left(1-\cos \frac{\eta}{R_{i}}\right)^{-3}
$$

where $M_{1}$ is a constant and $\overline{\Omega_{m}}(\eta)$ is the evolution of the matter density from the initial density.

Regarding the evolution of the Hubble parameter, by submitting Eq (12) to Eq (7) for nonrelativistic matter-dominated universe, we get:

$$
\dot{H}(\eta)=\frac{\ddot{a}}{a} \cong-\frac{32 \pi G M_{1}}{3 \Omega_{0, m}{ }^{3} R_{i}{ }^{6}}\left(1-\cos \frac{\eta}{R_{i}}\right)^{-3}
$$

By integrating, we get the expansion rate as follows: 


$$
H(\eta)=\frac{\dot{a}}{a} \cong \frac{8 \pi G M_{1}}{3 \Omega_{0, m}{ }^{3} R_{i}^{6}}\left(\frac{1}{5 \tan ^{5} \frac{\eta}{2 R_{i}}}+\frac{1}{3 \tan ^{3} \frac{\eta}{2 R_{i}}}+\frac{1}{\tan \frac{\eta}{2 R_{i}}}\right)+M_{2}
$$

where $M_{2}$ is a constant.

We can now trace the evolution of the spacetime worldlines in polar coordinate based on the spatial scalar factor and its evolution time starting from their minimum at the Big Bounce of the primordial substance of the minimal radius. The polar radius of the spacetime worldline $R_{L}(\eta)$ is:

$$
R_{L}(\eta)=\mp \sqrt{ }\left(c^{2} t(\eta)^{2}+a(\eta)^{2}\right)
$$

The $\mp$ solutions of the polar radius of the spacetime worldline evolution implies that the universe could be evolving in opposite directions where the negative radius solution could be an indication of the existence of the antimatter as a distinct side. Submitting Eqs $(9,11-14)$ to Eq (15), we get the polar radius of the spacetime worldline evolution:

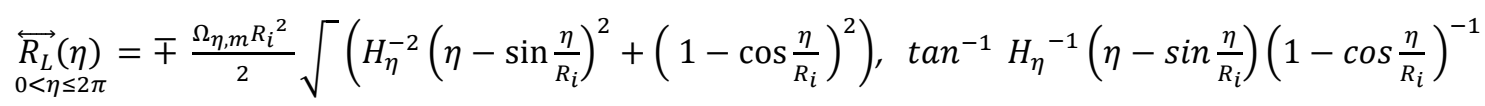

According to this, we can distinguish $R_{L}(\eta)$ which represents the spacetime worldline radius due to the age of the universe while $a(\eta)$ could represent a local radius of the universe slice at an instant of the time. $\overleftrightarrow{R_{L}}(\eta)$ can be considered as the cosmic horizon for an observer that is located at the minimal spatial and temporal origin.

\section{The Evolution of Spacetime Worldlines}

By tuning the integration constants of the derived model in Eq (16) to guide the mean evolution value of the Hubble parameter at around $\sim 70 \mathrm{~km} / \mathrm{s} / \mathrm{Mpc}$ and to guide a phase transition of expansion at an approximate universe age of $\sim 10 \mathrm{Gyr}$, the model predicted the density $\Omega_{0}=\sim 1.14$. In addition, the predicted spacetime worldlines of both sides are shown in Figure 1, where the cosmic evolution might experience three distinct phases.

Firstly, both sides of the universe expand away from the Big Bounce 'origin' which could be because of the power of the explosion and the electromagnetic fields that could throw the matter and antimatter in opposite directions. The slope of the worldlines during the first phase (the first $\sim 10$ Gyr) shows a deceleration until they reach their critical radii. The expansion rate is discussed in detail in the next Section.

However, at the second phase, the evolution worldlines reverse their directions and both sides free fall towards each other. The matter and antimatter could be under free-fall towards each other at gravitational acceleration, causing the current accelerated expansion of the universe. The slope of the worldlines during the second phase (after $\sim 10 \mathrm{Gyr}$ ) shows an accelerated rate. These two phases could be expressed as in the analog that when a ball is thrown upwards, it would decelerate until it stops and then it reverses its direction and free fall at an accelerated rate.

Interestingly, the model predicts a third phase of spatial contraction with a reversal-time arrow at 20 Gyr and afterwards due to a rapid surge in density i.e. drop in entropy according Eq (12). At this phase, the universe experiences a contraction which could be due to high concentration at both sides leading to a Big Crunch of a cyclic universe. 


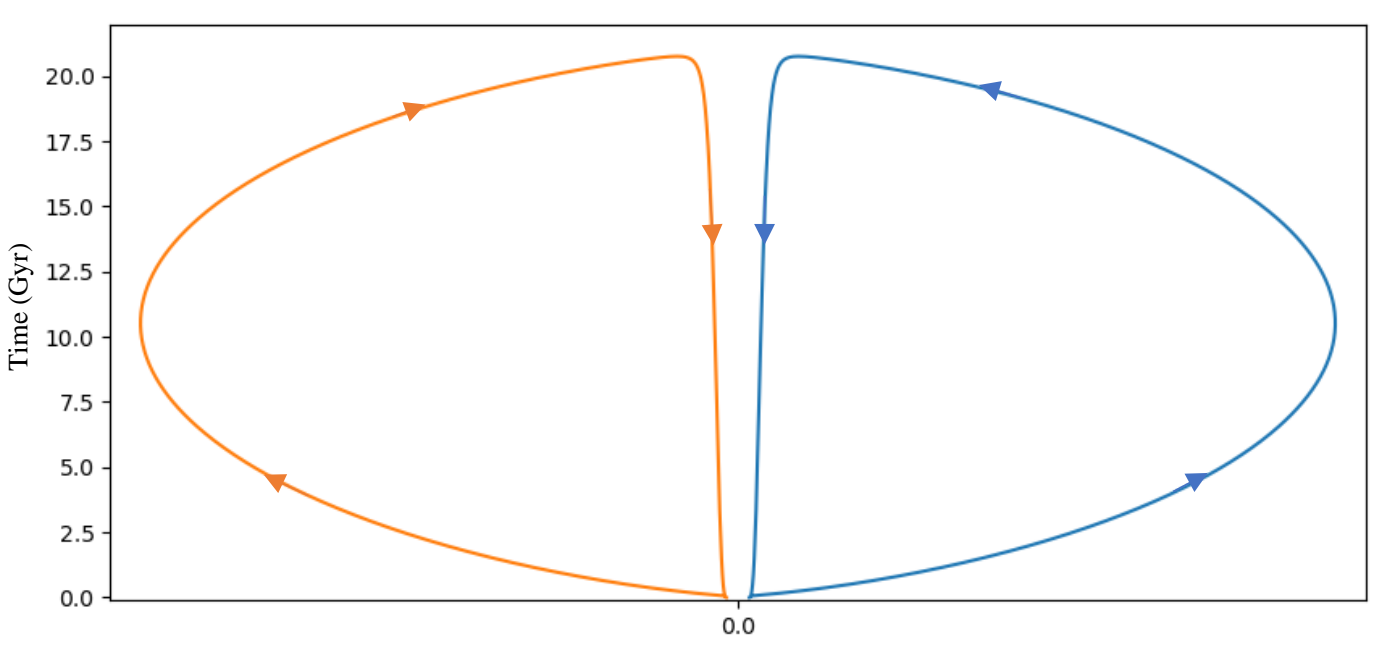

The evolution of spacetime worldlines

Figure 1. The worldlines of spacetime evolution of both sides, which are known to be unrestricted by the light worldlines. The spacetime worldlines are represented by the polar radius of the spatial scale factor and its evolution time. The evolutions are firstly a phase of spatial expansion away from the Big Bounce of both sides until they reach their critical radii. Then, a second spatial expansion phase in a reverse direction. Finally, a third rapid time-reversal phase of spatial contraction leading to a Big Crunch.

The worldlines of spacetime evolution of both sides can be schematically represented as shown in Figure 2. The first phase is shown in Figure 2-A where both sides expand in opposite directions away from the Big Bounce origin while Figure 2-B shows the second and third phases of reverse directions where both sides are free falling towards each other.

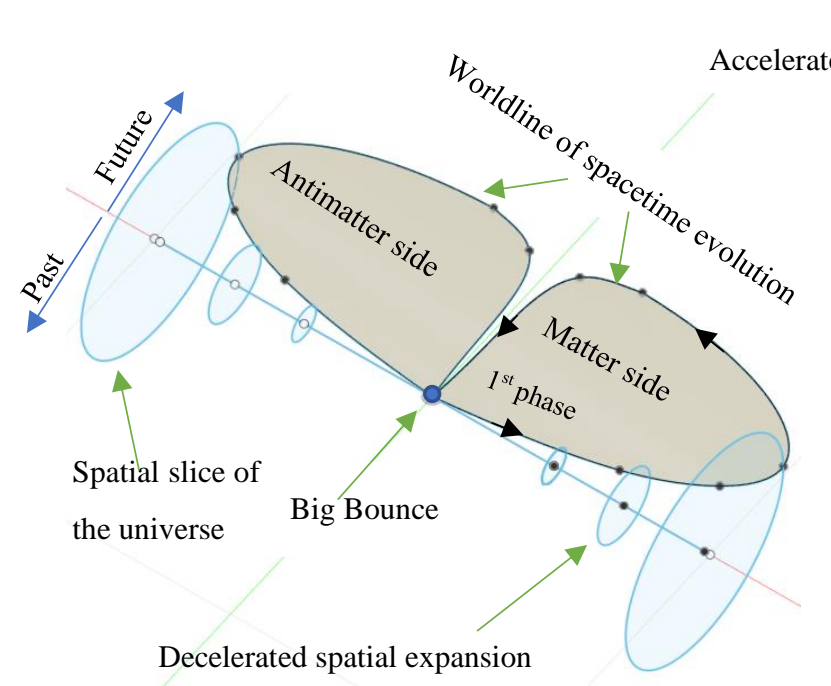

(A) First phase

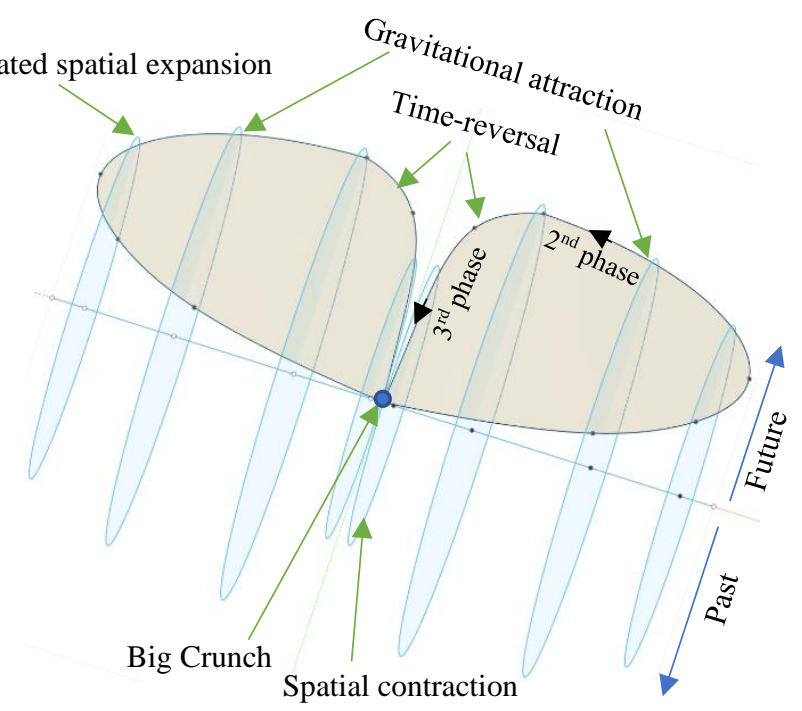

(B) Second and third phases

Figure 2. Schematic representation of both sides according the spacetime worldline evolution. (A) Both sides expand away from the Big Bounce origin. (B) At the second stage, both sides expand in reverse direction and free fall towards each other. In the third phase, both sides shrink which could be due to high concentration leading to a Big Crunch of a cyclic universe. Blue circles represent a 3-dimensional slice of the universe at an instant of time, which is not necessarily to be a simply path connected. 


\section{The Expansion and Flow Rates}

\subsection{Evolution of the Hubble Parameter}

The Hubble parameter or the speed of the spatial expansion $H=\dot{a} / a$ and its deceleration or acceleration rate $\dot{H}(\eta)=\ddot{a} / a$ can be found using Eq (14) and Eq (13) respectively. The predicted speed $H$, rectify speed, and deceleration/acceleration $\dot{H}$ of spatial expansion are shown in Figure 3. The Hubble parameter $H$ starts at its highest values at the Big Bounce, which could be as result of the power of the explosion where the speed of expansion at its highest value. Then the speed of expansion decreases during the first $10 \mathrm{Gyr}$, which could be due to gravity between the two sides, until it approaches its minimal value at the phase transition at around 10 Gyr.

Afterwards, the Hubble parameter starts to increases in a reverse direction (the minus sign at the second phase of the universe expansion speed confirms the opposite direction of the expansion direction discussed in the previous Section), which could be as a result that both sides free-fall towards each other at gravitational acceleration. Then it goes to its highest value at the Big Crunch.

According to the mechanics, the opposite signs of acceleration and expansion speed at first phase indicate a slowing down until the deceleration/ acceleration rate reaches its minimal value at around 10 Gyr while the same signs at the second phase indicates the expansion speed is increasing.

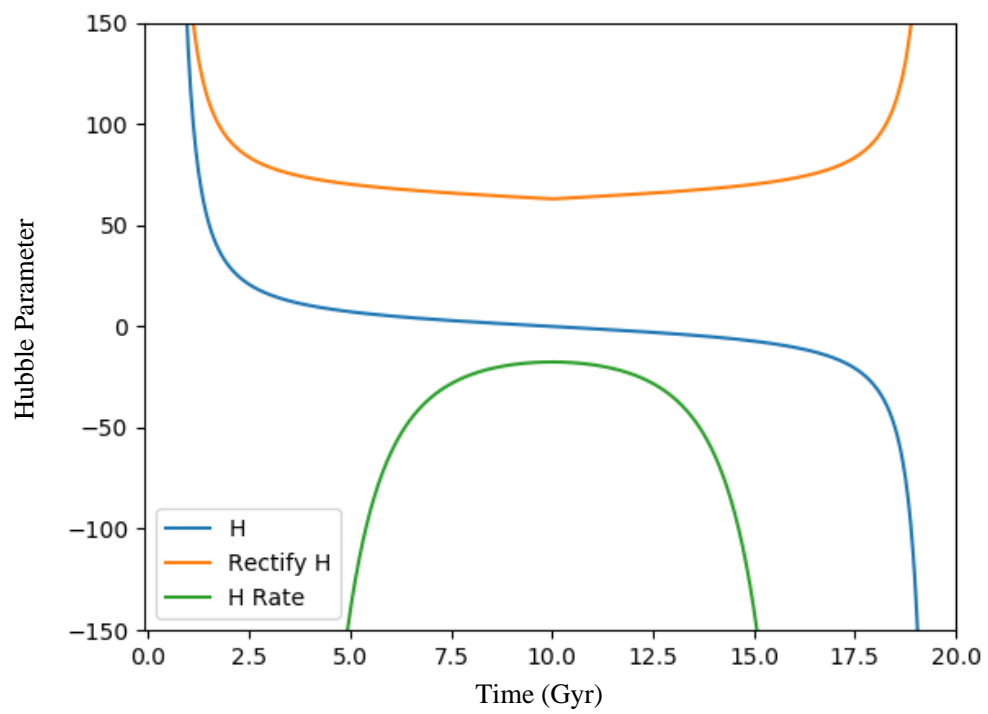

Figure 3. The Hubble parameter and its rate

The expansion rate was decreasing at the first phase, and this conforms with the lower value of the Hubble parameter obtained by the Planck datasets of the early universe. However, at the second phase, the expansion rate is increasing, and this aligns with the higher value of the Hubble parameter obtained using the supernovae type Ia distance-redshift method at the present universe.

\subsection{Flow Rate of Both Sides}

Both sides are predicted to expand away from each other during the first phase then they free fall towards each other at the second phase. The flow rate of the matter side of the universe due to the gravitational attraction by the antimatter side could be predicted by taking the derivative of Eq (16) with regards to the conformal time:

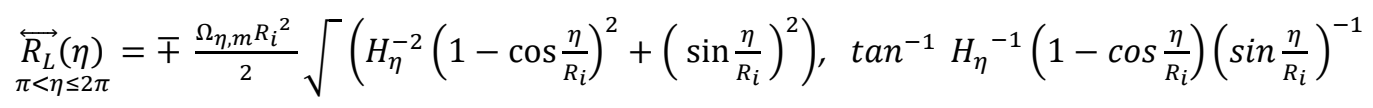




\section{Simulation of Spacetime Flatness and Voids}

The spacetime worldline of one side of the universe can be simulated according the derived model along with the nearer spacetime worldlines for both the early and present universe as shown in Figure 4A and 4B respectively. At the early universe as shown Figure 4-A, the evolution of the spacetime worldlines are not equal at any universe age. This means the spacetime is curved at the early universe in a similar shape of a ball surface and this aligns with recent findings of space curvature from $\mathrm{CMB}$ [27]. On the other hand, at present accelerated phase of expansion of reverse direction, the spacetime worldlines are aligned and they produce a flat spacetime as shown in Figure4-B. In addition, internal voids could be produced, which could continuously increase the matter and antimatter densities elsewhere in both sides.

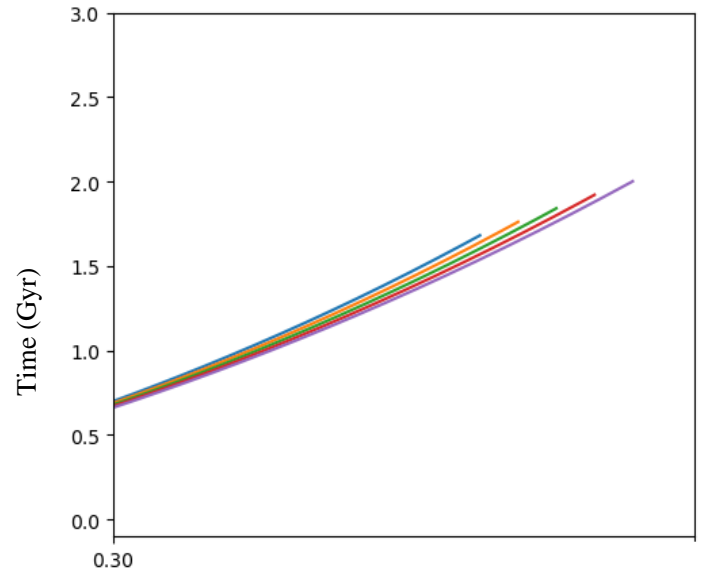

A) Spacetime worldlines at the early universe

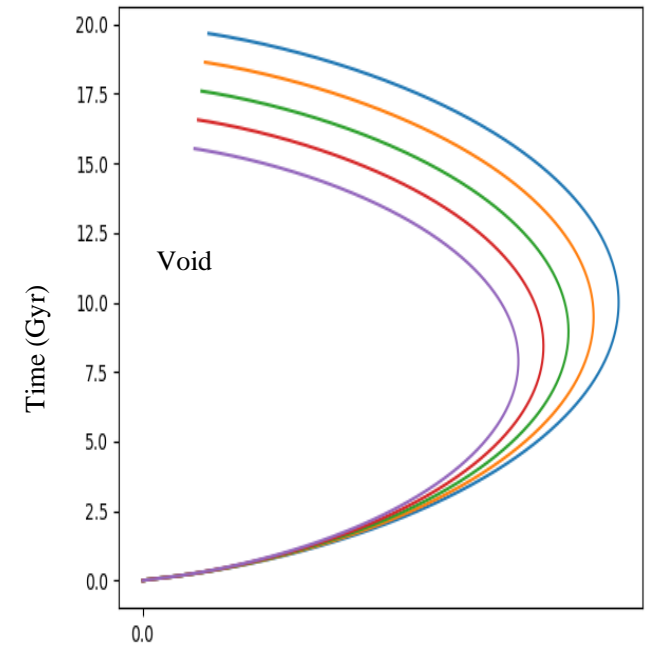

B) Spacetime worldlines

Figure 4. The evolution of spacetime worldlines at both early and present universe.

A schematic represented in 2D spatial and 1 temporal dimension is shown in Figure 5-A while Figure 5B shows the apparent topology due to gravitational lensing.

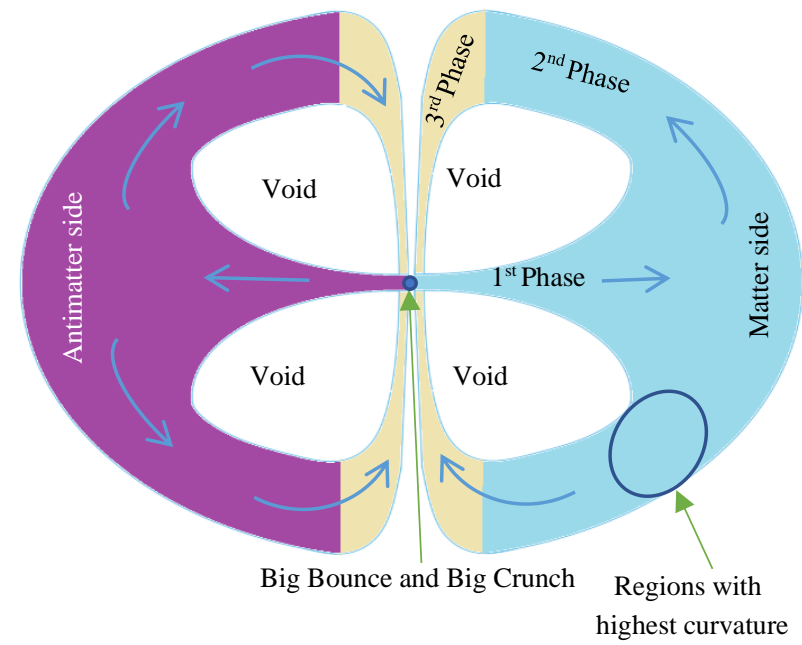

A: Cosmic topology

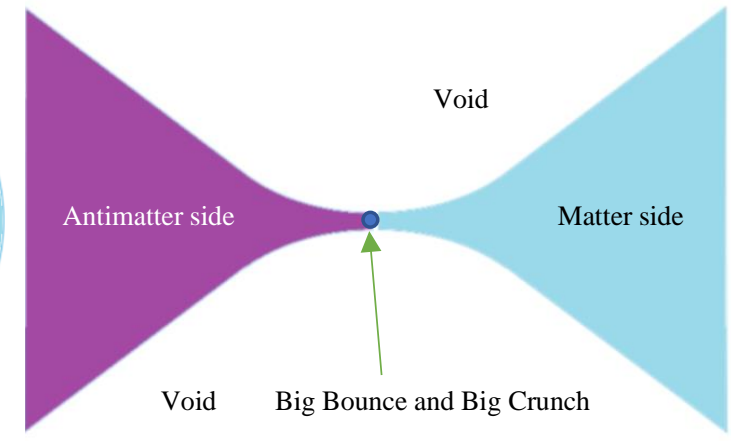

B: Apparent cosmic topology

Figure 5. 2D-Schematic representation of the predicted the cosmic topology of both sides of the universe where internal voids are predicted at the second phase of the reverse of direction. B) The apparent topology due to gravitational lensing of the universe during the first and second phases of expansions. 


\section{Simulation of Spiral Galaxy}

The consistent patterns of galactic rotation curves using precise and independent galactic redshift data confirmed that the hydrogen clouds and outer stars are orbiting galaxies at speeds faster than that calculated using Newtonian laws. Accordingly, the dark matter hypothesis was introduced to account for the apparently missing galactic mass and to explain the fast-orbital velocity [39], [40]. However, no evidence of the existence of the dark matter, which is supposed to account for the majority galactic mass, was observed since its introduction. The failure to find dark matter led to the introduction of new theories such as modified gravity and modified Newtonian dynamics [30], [31], [41]-[43]. On the other hand, several recent studies found that many galaxies do not contain dark matter [44], [45]. This observation was considered in some studies where the galaxy formation was simulated using modified Newtonian dynamics without considering the dark matter [32]. Thus, it seems that there is no evidence or agreement on the existence or nature of the dark matter as well as it is not an essential element in some galaxies.

As an alternative, as the predicted shape of spiral galaxies is very similar to a vortex shape, we can study a spiral galaxy as a forced vortex where an external torque is presence by the curvature of the spacetime worldlines along the evolution time. In Figure 5-A, it was shown that the curvature of the spacetime worldlines varies and increases along the evolution time with highest curvature at the phase transition. Accordingly, it can be purposed that the observed fast star orbital speeds could occur as a result of the variation of the universe curvature along the spacetime worldline.

To evaluate this, a fluid simulation study was performed based on the Newtonian dynamics using the Fluid - Pressure and Flow software [46]. In this simulation, a perfect fluid of mass density $\rho$ and isotropic pressure $p$ was assumed to represent the spacetime while the fluid flow was traced to study its movement. The fluid was considered as a perfect fluid because it is frictionless with no heat conductivity [47]. Using these conditions, the fluid model was built to simulate the fluid flow speed between an incrementing worldline curvature and their distance from a highly curved to a lesser spacetime curvature, as shown in Figure 6.

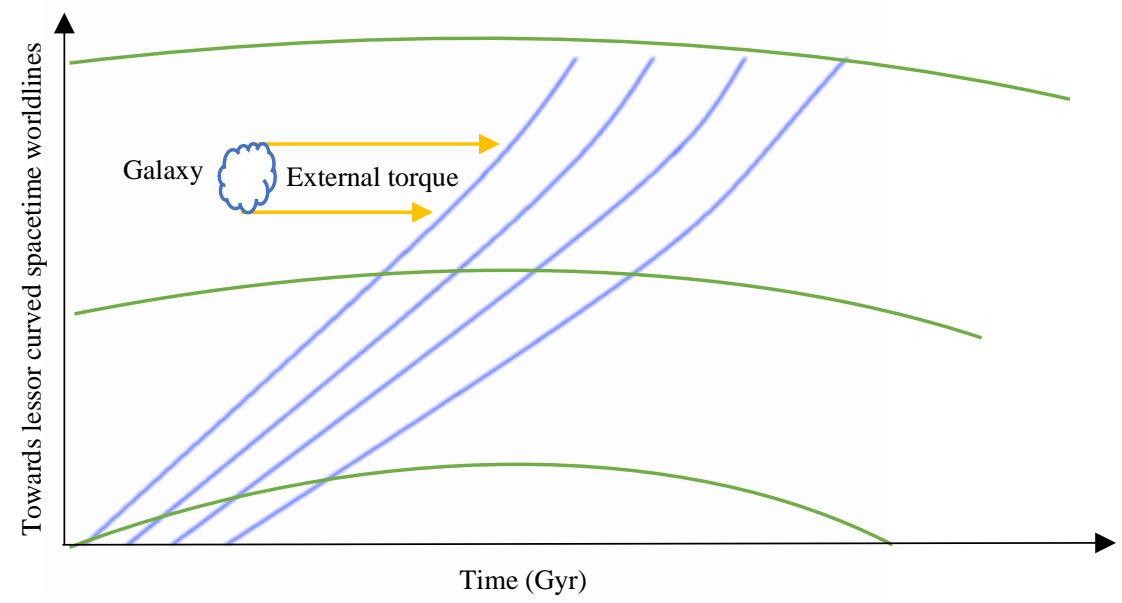

Figure 6. External torque exerted on a galaxy due to the divergence of the curvature of spacetime worldlines through time dimension. Green curves represent the curvature of spacetime worldlines. Blue curves represent the stimulated spacetime flux through the time dimension. 
Using the resultant torque, a spiral galaxy is simulated as a forced vortex as shown in Figure 7

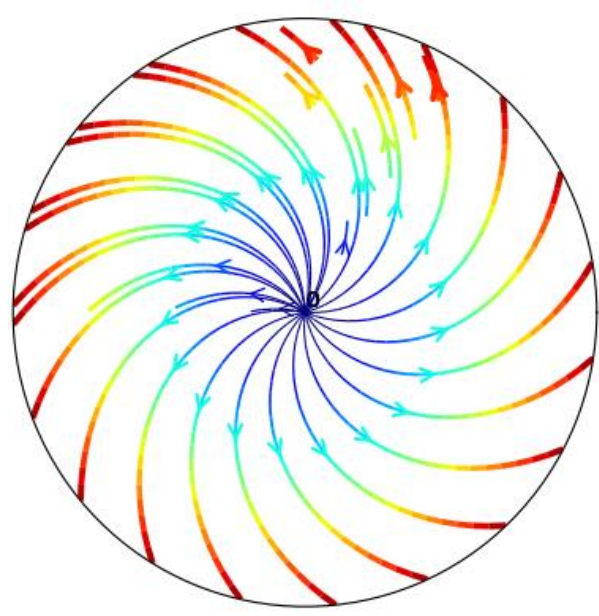

A) Spiral galaxy rotation at early universe

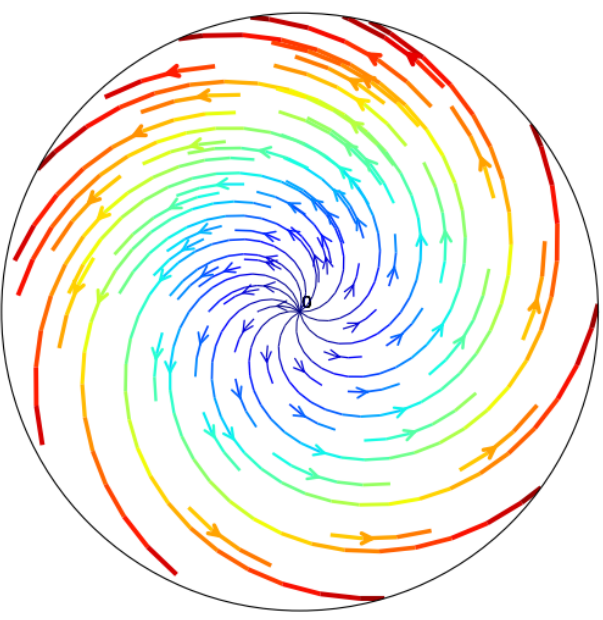

B) Spiral galaxy rotation at the present universe

Figure 7. Spiral galaxy rotation at early and present universe.

According to these outcomes, it could be concluded that the variation of the curvature of the over the time dimension could influence the speed galaxy rotation. These results seem to follow the TullyFisher relation.

\section{Conclusions}

In this research, I traced the evolution of the spacetime worldlines to construct a potential spatial curvature over the time dimension or a global spacetime curvature. The mathematical derivations of a positively curved universe governed by only gravity revealed two opposite solutions of the worldline evolution. It was concluded that these results imply the universe has two sides, matter and antimatter. The implementation the derived model, it predicted the density is $\Omega_{0}=\sim 1.14>1$. It also revealed a decelerated phase of spatial expansion during the first $\sim 10 \mathrm{Gyr}$, that is followed by a second phase of an accelerated expansion, which potentially matching the tension in Hubble parameter measurements. In addition, the model predicted a final time-reversal phase of spatial contraction leading to a Big Crunch of a cyclic universe. The rate during the early universe was decreasing while the rate at the current universe is increasing. These results conceivably align with Hubble parameter measurements from the early and current universe.

The abandonment of the cosmological constant in this model could fit the quantum field theory as it distinguishes the quantum vacuum energy from the energy of space and attributes the accelerated expansion to the gravitational attraction between both sides. However, the literature on dark matter/energy can be utilized to accurately estimate the matter and spatial density distribution.

Regarding the fast orbital speed of stars, the simulation can provide a plausible explanation where the curvature of spacetime through time dimension of both matter and antimatter sides could provide external torque on galaxies causing them to increase in speed over their evolution time, which seems to fit the Tully-Fisher relation.

These findings may indicate the existence of the antimatter as a distinct side, which influences the evolution of the universe instead of the dark energy or dark matter. 


\section{Future Work}

The research presented a new theory with promising outcomes and predictions, which can be verified, fine-tuned or disproved using astrometric data in future works. The integration constants of this model can be fine-tuned in order to accurately estimate of the matter and curvature densities. Finally, the accurate age of the universe could be estimated based on the non-linear Hubble parameter derived equation.

\section{References}

[1] V. Sahni, “The Cosmological Constant Problem and Quintessence,” Class. Quantum Gravity, vol. 19, no. 13, pp. 3435-3448, Feb. 2002.

[2] N. E. Mavromatos, “Models and (some) Searches for CPT Violation: From Early Universe to the Present Era," in Journal of Physics: Conference Series, 2017, vol. 873, no. 1.

[3] D. Sakharov, "Violation of $\mathrm{cp}$ in variance, C asymmetry, and baryon asymmetry of the universe," Sov. Phys. - Uspekhi, vol. 34, no. 5, pp. 392-393, May 1967.

[4] M. Ahmadi et al., "Investigation of the fine structure of antihydrogen," Nature, vol. 578, no. 7795, pp. 375-380, Feb. 2020.

[5] S. Eriksson, "Precision measurements on trapped antihydrogen in the ALPHA experiment," Philos. Trans. R. Soc. A Math. Phys. Eng. Sci., vol. 376, no. 2116, p. 20170268, Mar. 2018.

[6] J. . Gonzalo, Inflationary Cosmology Revisited: An Ove: An Overview of Contemporary Scientific Cosmology After the Inflationary Proposal. World Scientific, 2005.

[7] P. J. Steinhardt, "The inflation debate: Is the theory at the heart of modern cosmology deeply flawed?," Sci.Am., vol. 304N4, pp. 18-25, Apr. 2011.

[8] A. TRAUTMAN, “Spin and Torsion May Avert Gravitational Singularities,” Nat. Phys. Sci., vol. 242, no. 114, pp. 7-8, Mar. 1973.

[9] G. Unger and N. Popławski, "Big Bounce and Closed Universe from Spin and Torsion," Astrophys. Journal, 87078 (7pp), 2019, 2019.

[10] O. Klein, “Instead of cosmology,” Nature, vol. 211, no. 5056. Nature Publishing Group, pp. 1337-1341, 1966.

[11] O. Klein, “Arguments concerning relativity and cosmology," Science (80-. )., vol. 171, no. 3969, pp. 339345, Jan. 1971.

[12] G. Efstathiou, “Is the low cosmic microwave background quadrupole a signature of spatial curvature?,” 2003.

[13] D'Agostini G. and Petit J.P, “The Janus Cosmological Model. The radiative era as coupled gauge processes.," Astrophys. Space Sci., vol. 363, no. 139, 2018.

[14] S. J. Robles-Pérez, "Time reversal symmetry in cosmology and the creation of a universe-antiuniverse pair," Universe, vol. 5, no. 6, pp. 1-11, 2019.

[15] C. O'Raifeartaigh, “Historical and Philosophical Aspects of the Einstein World,” Jun. 2019.

[16] A. Friedman, “Über die Krümmung des Raumes,” Zeitschrift für Phys., vol. 10, no. 1, pp. 377-386, Dec. 1922.

[17] W. Xu, “Framework of Natural Cosmology," Virtumanity.

[18] S. Perlmutter et al., "Measurements of Omega and Lambda from 42 High-Redshift Supernovae," Astrophys. J., vol. 517, no. 2, pp. 565-586, Dec. 1998.

[19] A. G. Riess et al., “Observational Evidence from Supernovae for an Accelerating Universe and a 
Cosmological Constant," Astron. J., vol. 116, no. 3, pp. 1009-1038, Sep. 1998.

[20] A. G. Riess et al., "New Parallaxes of Galactic Cepheids from Spatially Scanning the Hubble Space Telescope: Implications for the Hubble Constant," Astrophys. J., vol. 855, no. 2, p. 136, Jan. 2018.

[21] A. G. Riess, "The expansion of the Universe is faster than expected," Nature Reviews Physics, vol. 2, no. 1. Springer Nature, pp. 10-12, 01-Jan-2020.

[22] P. J. E. Peebles and B. Ratra, “The cosmological constant and dark energy," Reviews of Modern Physics, vol. 75, no. 2. American Physical Society, pp. 559-606, 22-Apr-2003.

[23] E. Lusso et al., "Tension with the flat $\{\backslash$ Lambda $\} C D M$ model from a high redshift Hubble Diagram of supernovae, quasars and gamma-ray bursts," Astron. Astrophys., vol. 628, Jul. 2019.

[24] T. Josset, A. Perez, and D. Sudarsky, “Dark Energy from Violation of Energy Conservation," Phys. Rev. Lett., vol. 118, no. 2, p. 021102, Jan. 2017.

[25] R. J. Adler, B. Casey, and O. C. Jacob, “Vacuum catastrophe: An elementary exposition of the cosmological constant problem," Am. J. Phys., vol. 63, no. 7, pp. 620-626, Jul. 1995.

[26] E. Di Valentino, A. Melchiorri, and J. Silk, “Cosmic Discordance: Planck and luminosity distance data exclude LCDM," arXiv Prepr. arXiv2003.04935, Mar. 2020.

[27] E. Di Valentino, A. Melchiorri, and J. Silk, "Planck evidence for a closed Universe and a possible crisis for cosmology," Nat. Astron., vol. 4, no. 2, pp. 196-203, Feb. 2020.

[28] N. Aghanim et al., "Planck 2018 results. V. CMB power spectra and likelihoods," Astron. Astrophys., vol. 89, Jun. 2020.

[29] H. Hildebrandt et al., "KiDS-450: Cosmological parameter constraints from tomographic weak gravitational lensing," Mon. Not. R. Astron. Soc., vol. 465, no. 2, pp. 1-50, Feb. 2017.

[30] A. Maeder, "An alternative to the LCDM model: the case of scale invariance," Astrophys. J., vol. 834, no. 2, p. 194, Jan. 2017.

[31] M. M. Brouwer et al., "First test of Verlinde's theory of Emergent Gravity using Weak Gravitational Lensing measurements," Mon. Not. R. Astron. Soc., vol. 466, no. 3, pp. 2547-2559, Dec. 2016.

[32] N. Wittenburg, P. Kroupa, and B. Famaey, "The formation of exponential disk galaxies in MOND," Astrophys. J., vol. 890, no. 2, p. 173, Feb. 2020.

[33] K. L. Pandey, T. Karwal, and S. Das, "Alleviating the $\$$ H_0 $\$$ and $\$ \backslash$ sigma_8 $\$$ anomalies with a decaying dark matter model," Feb. 2019.

[34] N. Straumann, “General Relativity (Graduate Texts in Physics)," in Springer, Springer, 2013.

[35] M. Lachì Eze-Rey and J.-P. Luminet, “COSMIC TOPOLOGY,” arXivgr-qc/9605010v2 9 Jan 2003, 2003.

[36] G. F. R. Ellis and H. van Elst, “Cosmological models (Carg \’\{e\}se lectures 1998),” Dec. 1998.

[37] J. Fl, L. D. Landau, and E. Ml Lifshitz, “The Classical Theory of Fields Third Revised English Edition Course of Theoretical Physics," 1967.

[38] B. Ryden, Introduction to Cosmology. San Francisco, CA, USA: Addison Wesley, ISBN 0-8053-8912-1., 2006.

[39] P. D. Mannheim and D. Kazanas, "Exact vacuum solution to conformal Weyl gravity and galactic rotation curves," Astrophys. J., vol. 342, p. 635, Jul. 1989.

[40] Y. Sofue and V. Rubin, "Rotation Curves of Spiral Galaxies," Annu. Rev. Astron. Astrophys., vol. 39, no. 1, pp. 137-174, Oct. 2000.

[41] E. A. Chadwick, T. F. Hodgkinson, and G. S. McDonald, “Gravitational theoretical development supporting MOND," Phys. Rev. D - Part. Fields, Gravit. Cosmol., vol. 88, no. 2, p. 024036, Jul. 2013.

[42] J. R. Van Meter, “Dark-matter-like solutions to Einstein's unified field equations,” Phys. Rev. D, vol. 97, no. 4, p. 044018, Feb. 2018. 
[43] M. Milgrom, “MOND in galaxy groups: A superior sample,” Phys. Rev. D, vol. 99, no. 4, p. 044041, Feb. 2019.

[44] S. Dodelson and M. Liguori, “Can Cosmic Structure form without Dark Matter?,” Phys. Rev. Lett., vol. 97, no. 23, Aug. 2006.

[45] Q. Guo et al., "Further evidence for a population of dark-matter-deficient dwarf galaxies," Nat. Astron., vol. 4, no. 3, pp. 246-251, Jul. 2019.

[46] Sam Reid et al, "Fluid Pressure and Flow, PhET Interactive Simulations." University of Colorado, 2013.

[47] R. J. Nemiroff and B. Patla, "Adventures in Friedmann cosmology: A detailed expansion of the cosmological Friedmann equations," Am. J. Phys., vol. 76, no. 3, pp. 265-276, Mar. 2008. 